\title{
Proof of concept for adaptive sequential optimization of free-space laser communication receivers
}

\author{
Carlos E. Carrizo ${ }^{1,2, *}$, Ramon Mata Calvo ${ }^{1}$, and Aniceto Belmonte ${ }^{2}$ \\ ${ }^{1}$ Institute of Communications and Navigation, German Aerospace Center (DLR), Wessling, Germany, 82234 \\ ${ }^{2}$ Department of Signal Theory and Communications, Technical University of Catalonia BarcelonaTech, Barcelona, Spain, 08034 \\ *Corresponding author: carloscarrizo@gmail.com
}

Compiled February 11, 2019

\begin{abstract}
In a down-link scenario, performance of laser satellite communications is limited due to atmospheric turbulence, which causes fluctuations in the intensity and the phase of the received signal leading to an increase in bit error probability. In principle, a single-aperture phase-compensated receiver, based on adaptive optics, can overcome atmospheric limitations by adaptive tracking and correction of atmospherically induced aberrations. However, under strong-turbulence situations, the effectiveness of traditional adaptive optics systems is severely compromised. We have developed an alternative intensity-based technique that corrects the wave-front by iteratively updating the phases of individual focal-plane speckles, maximizing the power coupled into a single-mode fiber. Here, we present the proof of concept for this method. We show how this technique improves the quality of the received signal by $4 \mathrm{~dB}$ gain with less than 60 power measurements under strong turbulence conditions. ๑ 2019 Optical Society of America
\end{abstract}

OCIS codes: (110.0110) Adaptive Optics, Imaging through turbulent media; (030.0030) Speckle, Turbulence; (070.0070) Phase conjugation; (060.0060) Free-space optical communication.

http://dx.doi.org/10.1364/ao.XX.XXXXXX

\section{INTRODUCTION}

One of the main challenges in free-space optical communications is the compensation of the turbulence-induced wave-front distortions that deteriorate the quality of the received signal. When high data rates are intended in scenarios like satellite-to-ground downlinks, an efficient and stable single-mode fiber (SMF) coupling is required. These wave-front distortions induce strong fading in the coupled signal, resulting in unacceptable bit error rates (BER). Adaptive optics is generally adopted to minimize the impact of the atmosphere. Real-time systems, like those based on Shack-Hartmann wave-front sensor, are very efficient as long as the turbulence is weak-to-moderate [1]. For stronger turbulence conditions, scintillation and phase singularities limit its performance, and the wave-front correction may be better faced with iterative systems [2].

Generally, iterative methods operate on the phase compensator element, typically a deformable mirror (DM), modifying its shape stochastically while trying to optimize a performance metric, like power in the bucket. The drawback of these systems is the correction time, which depends on the total number of required iterations. This time is commonly much larger than the coherence time of the field which, for LEO-satellite-downlinks, it is also influenced by the beam angular velocity due to the satellite displacement. Overall, the available time for a phase wave-front correction can be rounded to 1 milliseconds $[3,4]$. Considering that existing iterative solutions need more than 100 iterations to converge to an optimum, we would require unpractical loop bandwidths on the order of $100 \mathrm{kHz}[2,5]$. An alternative is a hybrid system which combines real-time wavefront sensors to provide a system hot start, effectively reducing the number of iterations [6]. However, hardware complexity increases and functional limitations of the complementary sensor may be inherited.

As a result of these limitations, it is of high interest to efficiently compensate the field with minimized bandwidth utilization. For such a goal, we have proposed a sequential optimization technique based on the signal processing of the speckle pattern in the focal plane [7]. It works by iteratively updating and optimizing the phases related to individual speckles, maximizing the composite signal with a number of iterations proportional to the number of treated speckles.

The objective of this paper is to validate in the laboratory the theoretical concept and numerical analyses shown in [7]. We aim to demonstrate that this technique increases the mean coupled power and reduces its variance, minimizing the overall bandwidth utilization.

The remainder of this paper is organized as follows. Section 2 defines the expected turbulent regimes of a LEO downlink and principal metrics to evaluate the $\mathrm{AO}$ system performance. 
Section 3 briefly explain the working principle of the adaptation method. The experimental setup is detailed in section 4 . The measurement procedure is explained in section 5 . Results are presented and discussed in section 6 . Section 7 concludes this paper.

\section{LEO DOWNLINK SCENARIO}

A laser downlink from a LEO satellite is affected differently by the turbulence when the elevation changes. In the atmosphere, random microscopic temperature fluctuations at different altitudes modify the refractive index of the media, introducing inhomogeneities that alter the wave-front characteristics [8]. The degree of the wave-front deterioration is related to the cumulative effect of the inhomogeneities alongside the optical trajectory, thus, longer paths represent the worst scenario. The AO compensation system must work efficiently over all these turbulent regimes. Four important metrics define the $\mathrm{AO}$ system for laser communications. By profiling the turbulence, the spatial coherence of the wave-front indicates the degree of amplitude and phase distortions. Amplitude distortions, leading to scintillation effects, are compensated with aperture averaging. Phase distortions are dominant and directly impact in the efficiency of the coupled signal. The temporal coherence defines the bandwidth of the compensation system.

\section{A. Turbulence strength}

The turbulence strength is profiled over the height $h$ with the index of refraction structure parameter $C_{n}^{2}(h)$. This parameter is measured alongside the optical path based upon the location of the optical ground station (OGS), resulting in several models adapted to the particular conditions of the place. The most popular model is the Hufnagel-Valley (HV)

$$
\begin{aligned}
C_{n}^{2}(h)= & 0.00594\left(v_{0} / 27\right)^{2}\left(10^{-5} h\right)^{10} \exp (-h / 1000) \\
& +2.7 \times 10^{-16} \exp (-h / 1500)+A \exp (-h / 100),
\end{aligned}
$$

where $h$ is in meters, $v_{0}$ is the rms wind speed in meters per second, and $A$ is the nominal value of $C_{n}^{2}(0)$ at the ground in $\mathrm{m}^{-2 / 3}$. The $v_{0}$ is calculated as

$$
v_{0}=\left[\frac{1}{15 \times 10^{3}} \int_{5 \times 10^{3}}^{20 \times 10^{3}} V^{2}(h) d h\right]^{1 / 2},
$$

where $V^{2}(h)$ is described by the Bufton wind model,

$$
V(h)=v_{\mathrm{s}} h+v_{\mathrm{g}}+30 \exp \left[-\left(\frac{h-9400}{4800}\right)^{2}\right] .
$$

Here, $v_{\mathrm{g}}$ is the wind speed at ground level, and $v_{\mathrm{s}}$ represents the slew rate due to the satellite displacement respect to the ground station,

$$
\begin{gathered}
v_{\mathrm{s}}=\frac{V_{\text {sat }}}{L} \frac{L^{2}+H_{\text {sat }}^{2}+2 R_{\mathrm{e}} H_{\text {sat }}}{2 L\left(H_{\text {sat }}+R_{\mathrm{e}}\right)} \\
L(\alpha)=\left[\left(H_{\text {sat }}+R_{\mathrm{e}}\right)^{2}-R_{\mathrm{e}}^{2} \cos ^{2}(\alpha)\right]^{1 / 2}-R_{\mathrm{e}} \sin (\alpha)
\end{gathered}
$$

The calculation of $v_{s}$ requires the satellite velocity $V_{\text {sat }}$, satellite altitude above the ground station $H_{\text {sat }}$, and the earth radius $R_{e}=6378 \mathrm{Km}$. Based on the link elevation $\alpha$ [rad], the satellite distance $L$ is approximated. Typical values for a satellite-toground link in daylight conditions are $A=1.7 \times 10^{-14} \mathrm{~m}^{-2 / 3}$, ground wind $v_{\mathrm{g}}=8[\mathrm{~m} / \mathrm{s}]$, satellite velocity $V_{\text {sat }}=7[\mathrm{~km} / \mathrm{s}]$, and $H_{\text {sat }}=510 \mathrm{Km}$.

\section{B. Fried parameter}

The atmospheric coherence width, commonly known as Fried parameter $r_{0}$, is a widely adopted metric to evaluate the degree of distortion of the wave-front. It measures the coherence of the beam transversely and has units of length usually expressed in centimeters. In practice, it defines the diameter of a circular area over which the rms wave-front aberration is equal to 1 radian.

The $r_{0}$ is approximated by integrating over the $C_{n}^{2}(h)$ profile for a particular distance between the ground station altitude $h_{0}$ and the satellite altitude $H=L(\alpha) \cos (\zeta)$, where $\zeta=\pi / 2-$ $\alpha[\mathrm{rad}]$ is the the zenith angle, and the wavelength of the light source $\lambda$ is implicit in the wave number $k=2 \pi / \lambda$.

$$
r_{0}=\left[0.423 k^{2} \sec (\zeta) \int_{h_{0}}^{H} C_{n}^{2}(h) d h\right]^{-3 / 5}
$$

In Eq. (6), the $r_{0}$ increases proportionally to $\lambda^{6 / 5}$ of the laser beam and decreases with the zenith angle.

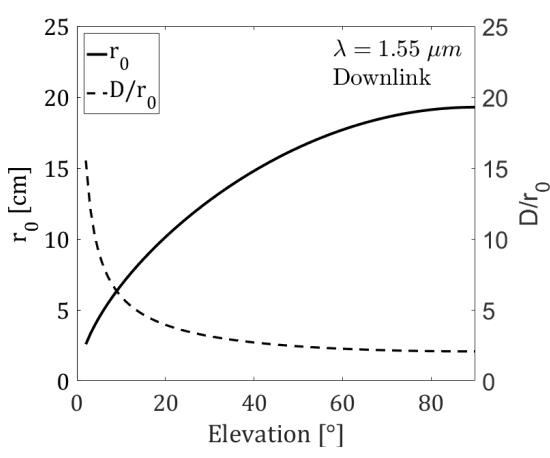

Fig. 1. Fried parameter and normalized aperture for a range of LEO-downlink elevations, considering a laser wavelength $\lambda=1.55 \mu \mathrm{m}$, an rms wind $v_{0}=21 \mathrm{~ms}$, and $D=40 \mathrm{~cm}$.

In laser communications, a widely used metric is the normalized aperture $D / r_{0}$. This metric can be used to approximate the number of dispersed power spots $M \approx\left(D / r_{0}\right)^{2}$ of a focused beam, viewed as focal intensity speckles. The higher the $D / r_{0}$, the more disperse the focal energy and thus more difficult it is to couple the light into a fiber. Figure. 1 shows the values of $r_{0}$ and $D / r_{0}$ for a LEO downlink at different satellite elevations and for a telescope aperture $D=40 \mathrm{~cm}$.

\section{Coherence time}

The coherence time of the field $\tau_{0}$ is an important design parameter of a wave-front compensation system. This parameter represents the time scale over which the phase wave-front can be considered to remain static, thus, it defines the available time for the system to compensate the distorted beam. The field can be considered partially coherent within $\tau_{0}$ and uncorrelated for a time $t>\tau_{0}$. The reciprocal of $\tau_{0}$ is the Greenwood frequency or bandwidth required for the compensation system.

The $\tau_{0}$ can be approximated with the $r_{0}$ and the rms wind speed $v_{0}$ as [9? ]

$$
\tau_{0} \approx \frac{0.314 r_{0}}{v_{0}}
$$

As shown in Fig. 2, the expected time scale of $\tau_{0}$ for laser downlinks in a moving satellite, ranges between 1.5 and 0.5 milliseconds for an increasing link elevation [3]. Note that the $\tau_{0}$ reduces towards the zenith due to the increment in the relative velocity of the beam. 


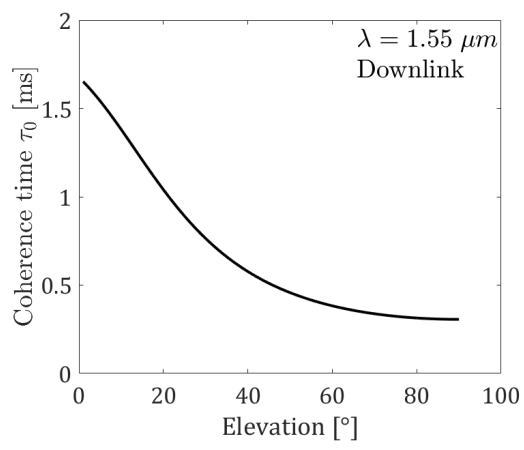

Fig. 2. Coherence time for a range of LEO-downlink elevations, considering a laser wavelength $\lambda=1.55 \mu \mathrm{m}$, and rms wind $v_{0}$ affected by the satellite slew rate.

\section{Fiber coupling}

A stable and efficient SMF coupling is essential to achieve multigigabit throughput. It allows the integration of optical amplifiers and coherent receivers. The SMF coupling efficiency $\eta$ can be generalized in function of the $D / r_{0}$, for plane wave and Kolmogorov spectrum, as [10-13]

$\eta=8 a^{2} \iint_{0}^{1} \exp \left[\frac{-\left(a^{2}+\left(\frac{1.048 D}{r_{0}}\right)^{2}\right)}{\left(x^{2}+y^{2}\right)^{-1}}\right] \frac{I_{0}\left(\left(\frac{1.48 D}{r_{0}}\right)^{2} x y\right)}{(x y)^{-1}} d x d y$

where $I_{0}$ is the modified Bessel function of first kind and zero order, which results from the back transformation of the fundamental fiber mode from the focal plane to the pupil plane. The parameter $a$ is a design coefficient relating the radius of the pupil $D / 2$ to the radius of the fiber core, and is set equal to 1.12 to achieve maximum coupling efficiency.

Synthetically generated fields are required for numerical simulations and laboratory tests. Here, the SMF coupling efficiency $\eta$ is calculated with the overlap integral between the complex pupil field $\boldsymbol{P}_{c}$, and the back propagated fundamental mode of the fiber $\boldsymbol{U}_{0}[13]$

$$
\eta=\frac{\left|\int \boldsymbol{P}_{c}^{*}(r) \boldsymbol{U}_{0}(r) \mathrm{ds}\right|^{2}}{\int\left|\boldsymbol{P}_{c}(r)\right|^{2} \mathrm{ds} \cdot \int\left|\boldsymbol{U}_{0}(r)\right|^{2} \mathrm{ds}} .
$$

In Eq. (9), the complex conjugate is indicated by $*$ and $r$ is the radial distance. The power-normalized, back propagated mode of a single-mode fiber with $1 / e$ mode field radius $w_{0}$, is calculated as

$$
\boldsymbol{U}_{0}=\sqrt{\frac{2}{\pi w_{\mathrm{a}}^{2}}} \exp \left(-\frac{r^{2}}{w_{\mathrm{a}}^{2}}\right)
$$

where $w_{\mathrm{a}}=\lambda f /\left(\pi w_{0}\right)$ is the back propagated mode field radius of the fiber, $\lambda$ is the laser wavelength, and $f$ is the focal length of the coupling lens. The maximum possible SMF coupling efficiency, assuming an ideal plane wave in the aperture, is $\eta=0.814$.

Performance curves of both approaches are later contrasted against laboratory measurements to evaluate the proper generation of turbulent scenarios.

\section{AO WORKING PRINCIPLE}

The method considers that the distorted pupil phase wave-front $\varphi$ can be approximated by a combined set of $M$ weighted plane wave modes, each one associated to one of the $M$ focal speckles of higher intensity. A simplified schematic of this method is shown in Fig. 3.

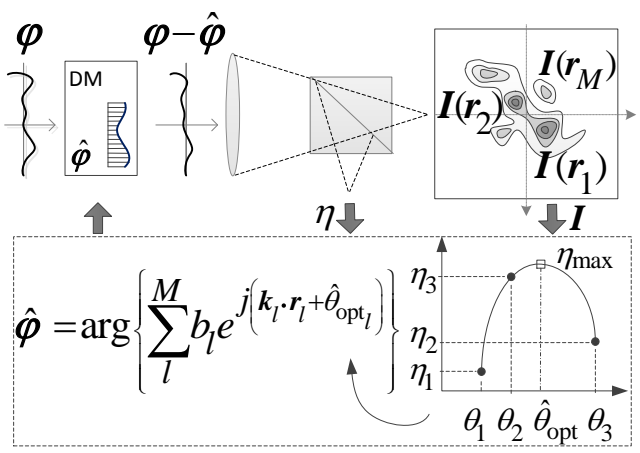

Fig. 3. Simplified schematic of the method

A focal camera is used to capture the speckle pattern, image processing is applied to identify and classify the focal speckles, and geometrical optics is used to define the correspondent pupil plane waves. The $l$ th plane wave is defined by its propagation vector $\boldsymbol{k}_{l}$ and the coordinate position $\boldsymbol{r}_{l}$ of the associated speckle maximum in the focal plane. The intensity level $b_{l}$ of each plane wave is calculated as the normalized amplitude of the associated speckle peak at the focal coordinates $\boldsymbol{r}_{l}$ in the camera sensor. Then, the optimal phase shape of the DM can be estimated as [7]

$$
\hat{\boldsymbol{\varphi}}=\arg \left\{b_{1} \exp \left(j \boldsymbol{k}_{1} \cdot \boldsymbol{r}_{1}\right)+\sum_{l=2}^{M}\left[b_{l} \exp \left(j\left(\boldsymbol{k}_{l} \cdot \boldsymbol{r}_{l}+\theta_{l}\right)\right)\right]\right\} .
$$

Here, the iterative phase compensation method follows a coordinate-wise ascent algorithm that treats the multivariate problem as a set of simpler sub-problems of a single dimension, maximizing the objective function $\eta$ by optimizing the scalar variables $\theta_{l}$ in sequence. The first term in Eq. (11) represents the first plane wave that acts as a reference from which the estimated field will evolve; thus, no phase shift is required. By starting with the second plane wave $l=2$, its optimum phase shift $\hat{\theta}_{\text {opt } l}$ is discovered following a parabolic optimization process $[14,15]$. In this process, three scalar phase variations $\left[\theta_{1}, \theta_{2}, \theta_{3}\right]$ are applied to the plane wave, shaping the mirror with three resulting estimations $\left[\hat{\varphi}_{1}, \hat{\varphi}_{2}, \hat{\varphi}_{3}\right]$. Concurrently, power measurements $\left[\eta_{1}, \eta_{2}, \eta_{3}\right]$ are made to finally find the optimum phase shift $\hat{\theta}_{\text {optl }}$ for the maximum coupling $\eta_{\max }$ of a parabolicfitted curve. This process is repeated for each subsequent plane wave, keeping each previously optimized variable fixed. The compensation of a distorted phase wave-front is done with a total number of iterations equal to

$$
N_{\text {iter }}=3(M-1)+1
$$

which is directly proportional to the number of main intensity speckles. A detailed explanation of each step can be found in [7].

\section{EXPERIMENTAL SETUP}

The schematic of the optical design and the image of the setup for the validation of the method are shown in Fig. 4 (a) and Fig. 4 (b), respectively. The relevant hardware components are listed in table 1 . The trajectory of the laser beam is represented with the arrows in Fig. 4 (b). Following the path, a continuous 
(a)

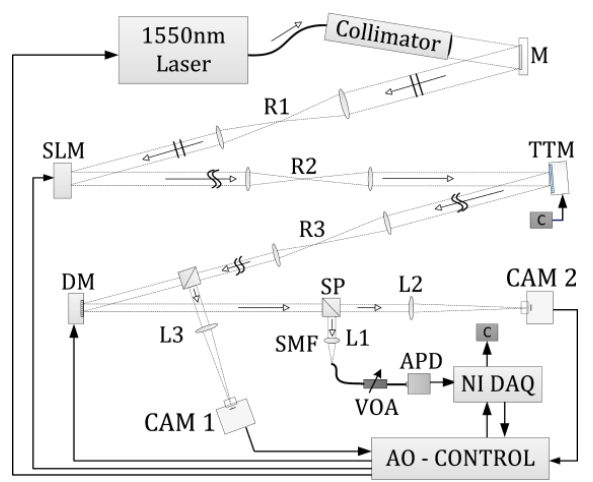

(b)

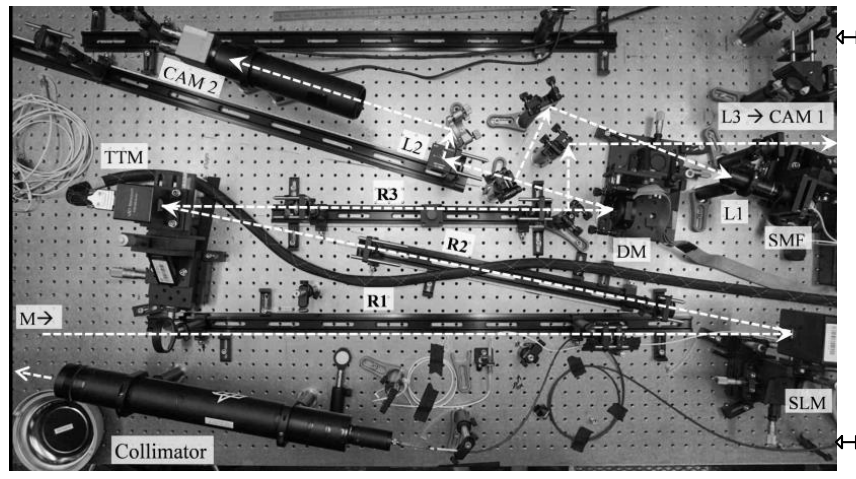

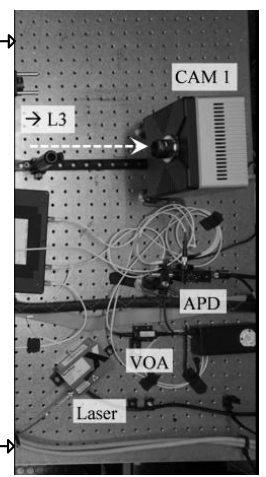

Fig. 4. (a) Optical receiver with the main hardware components. (b) Prototype of the receiver. The labels indicate the main components, namely: relay systems (R), Lenses (L), Splitters (Sp), Mirrors (M), Spatial Light Modulator (SLM), deformable mirror (DM), tip-tilt mirror (TTM), single mode fiber coupling (SMF), and infrared camera (CAM). The arrows show the optical path.

\begin{tabular}{l|l|l}
\hline Hardware & \multicolumn{1}{|c|}{ Type } & \multicolumn{1}{c}{ Details } \\
\hline SLM & Hamamatsu X10468-LCOS & $\begin{array}{l}\text { Windows }=15.8 \times 12 \mathrm{~mm} \\
800 \times 600 \text { pixels } \mid 20 \mu \mathrm{m} \text { pixel pitch } \\
\text { Resolution }=25 \mathrm{lp} / \mathrm{mm}\end{array}$ \\
\hline TTM & Newport FSM-300 & Closed-loop $600 \mathrm{~Hz}$ \\
\hline CAM 1 & Xenics Cheetah 640-CL & $\begin{array}{l}\text { InGaAs sensor } 640 \times 512 \text { pixels } \\
\text { Pixel Size } 20 \mu m \\
\text { Frame rate }=400 \mathrm{~Hz} \text { (Full) }\end{array}$ \\
\hline CAM 2 & Xenics Xeva XS-1.7-320 & $\begin{array}{l}\text { InGaAs sensor } 320 \times 256 \text { pixels } \\
\text { Pixel Size } 30 \mu m \\
\text { Frame rate }=100 \mathrm{~Hz} \text { (Full) }\end{array}$ \\
\hline DM & BMC Multi-X-CL140-DM & $\begin{array}{l}\text { Membrane I } 12 \times 12 \text { actuators } \\
\text { Max. Stroke }=3.7 \mu m \\
\text { Mirror BW }=3.5 \mathrm{KHz}\end{array}$ \\
\hline APD & Thorlabs PDA20CS-EC & InGaAs \\
\hline DAQ & NI BNC-2110 I PCI-6221 & 12 bits \\
\hline VOA & Thorlabs VOA50 & $50 \mathrm{~dB}$ \\
\hline
\end{tabular}

Table 1. List of hardware components of the optical setup.

laser $(1550 \mathrm{~nm})$ is collimated to generate a free-space laser beam with a diameter of $50 \mathrm{~mm}$. The beam size is modified throughout the system using a set of three optical relay systems (R1-3). After the first relay (R1), the beam is reflected in a spatial light modulator (SLM) to impress the phase-only distortions in the wave-front. The SLM can modulate the phase wave-front with a maximum phase excursion of $2 \pi$. Then, the distorted beam is guided to a tip-tilt mirror (TTM) to correct the phase-front tilt. After that, the beam is newly compressed with the relay (R3) to a diameter of $3.3 \mathrm{~mm}$ and split. One beam is focused in an infrared camera (CAM 1) with a $500 \mathrm{~mm}$ lens (L3). This focal camera is used for the optimization process, and it is located before the $\mathrm{DM}$ to register each speckle pattern before compensation. The second beam is reflected in a membrane-based DM, illuminating a section of $10 \times 10$ actuators. Here, the measured effective maximum stroke is $2.8 \mu \mathrm{m}$. Finally, the light beam is split and focused in an infrared camera (CAM 2) and a single mode fiber (SMF). For the camera, a lens (L2) of $500 \mathrm{~mm}$ is used to assure sufficient speckle resolution. This camera is used to verify the evolution of the focal speckle pattern during the correction process. For the SMF, a lens (L1) of $19 \mathrm{~mm}$ is used, achieving a maximum coupling efficiency of $70 \%$ when the wave-front is assumed aberration free. The signal for the optimization process is measured with an avalanche photodetector (APD). The coupled power can be attenuated with a single mode variable optical attenuator (VOA). The output electrical signal from the photodetector is acquired with a data acquisition system (DAQ).

\section{MEASUREMENTS PROCEDURE}

The proof of concept experiments is performed in a non-real-time regime. The algorithm, as well as the acquisition and control of all the described hardware, is implemented in Matlab. As previously mentioned, beam shaping is performed with a phasemodulation SLM to emulate the turbulent effects in the laser wave-front. The phases are created using a single-screen phase method [16], which allows fast generation of a large number of statistically uncorrelated wave-fronts. In this method, a set of weighted Zernike polynomials is combined. For the weights, an equal number of Karhunen-Loève coefficients is generated using the diagonalized covariance matrix of the Zernike polynomials. We generate each phase wave-front with 600 Zernike polynomials.

Prior to the compensation process, the system must be calibrated. A set of all possible tilted phases required for the software-generated plane waves is stored in a lookup table. As shown in Fig. 5, these phases are discovered by mapping each pixel coordinates of the camera sensor with a unique DM tilt using a mirror scanning process. The active scanned area is directly related to the stroke capacity of the DM and the design of the optical system.

During the compensation process, each phase screen is applied to the SLM, the overall phase wave-front tilt is corrected with the TTM, and the focal image of size $160 \times 160$ pixels is acquired. The speckles are detected using a local maxima algorithm which is fast and efficient [17]. With the identified speckles, the lookup table is used to generate the proper plane waves. Note that the speckles inside the active area can be assigned to a plane wave as the phase map is available. The speckles outside the active area are discarded. To continue, the algorithm performs the sequential iterative DM shaping, signal power acquisition, and phase optimization. This process is repeated for all the set of phase screens. For each case, the initial 
and final coupled power as well as the signal on each iteration and corrected speckle is registered.

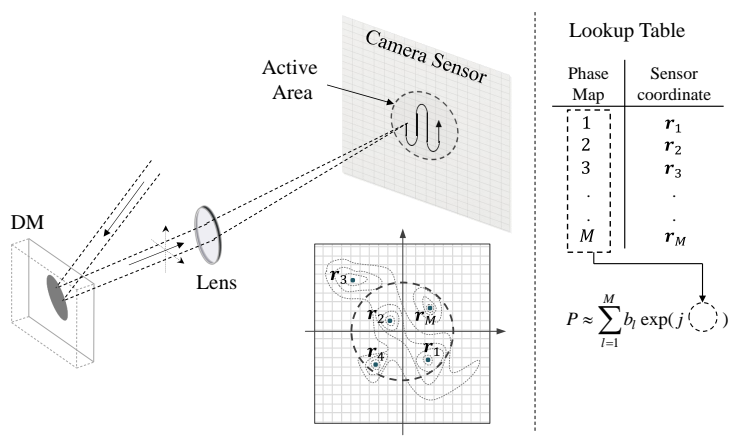

Fig. 5. Process to create the lookup table. The DM scans the camera sensor applying phase tilts to the reflected beam. The position of the speckle peak in the sensor is registered.

An evaluation of the AO system for a LEO-downlink requires a proper emulation of the turbulent scenarios. Figure 6 shows the mean SMF coupling penalty, normalized to the maximum $\eta$, for regimes between weak $D / r_{0}=1$ and strong $D / r_{0}=6$ turbulences, and for elevations ranging from $90^{\circ}$ to $10^{\circ}$, respectively. This range is selected based on the calculations in Fig. 1. The simulation curve (SIM 1) is performed with the synthetically generated fields and Eq. (9). The simulation curve (SIM 2) is calculated with the expected $D / r_{0}$ and Eq. (8). The markers correspond to the measured coupling penalty for each set of phase-screens, flat DM, and no-tilt correction. The good match between simulation and laboratory results verifies a correct generation of the different turbulent regimes.

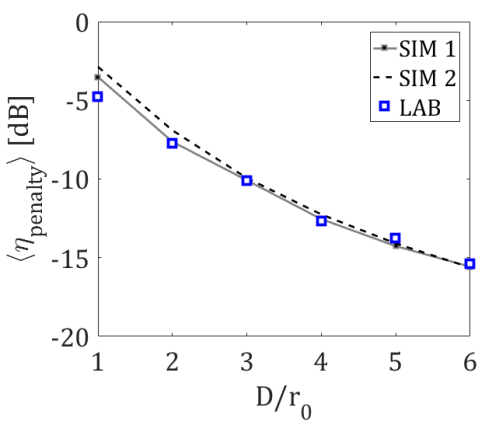

Fig. 6. Verification of synthetically generated fields through a comparison of the SMF coupling penalty.

To validate the method, we firstly evaluate the behavior of the algorithm with varying received signal power under the effects of noise. Then, we verify the overall performance over a range of turbulent scenarios and reduced bandwidth utilization.

\section{RESULTS}

Figure 7 (a) depicts the mean SMF coupling efficiency penalty for each corrected speckle in a scenario of strong turbulence. A total of 500 fields are created for a normalized aperture $D / r_{0}=6$, where $D$ is the beam diameter and $r_{0}$ indicates the spatial coherence of the wave-front. This case is selected to be representative of a LEO satellite downlink below $10^{\circ}$ elevation when the beam is received with a telescope aperture of $D=40 \mathrm{~cm}[4,18]$. The experimental values are normalized to the maximum achievable coupled power. Since the fluctuation of the acquired signal directly impacts the optimization process of each plane wave, the analysis is performed for values of $\mathrm{SNR} \approx[14,40]$ and received powers of $-60 \mathrm{dBm}$ and $-36 \mathrm{dBm}$, respectively. This range of received power is representative for multi-gigabit data reception, either working with direct detection or coherent systems $[19,20]$, and it corresponds for the APD power range between low and high SNR where the noise has minimal impact in the optimization process.

As expected from our initial numerical analyses [7], with higher SNR the coupling improves with each optimization, delivering a gain of after approximately 60 iterations. The inset shows the probability density functions (PDFs) of the final coupled signal, with a noticeable reduction in the variance compared with the tilt-corrected cases. The performance penalty due to the decrease in the SNR is also verified. The system can still provide a gain of $3 \mathrm{~dB}$ with 60 iterations. Here, the noise introduces random deviations in the measured power values used for optimization, affecting the parabolic fitting and leading to sub-optimum phase shift estimations. This effect is prone to impact more in speckles of lower intensity when the SNR is high, limiting the slope of the curve. Also, it can alter the initially estimated phase shift associated with speckles of higher intensity, forcing an earlier saturation. In Fig. 7 (b) the evolution of the coupling penalty against the mean SNR is depicted. The right axis is associated with the PDFs of each SNR case, which illustrate the noise distribution when the optical power varies during the iterative process. The right-skewed shape of the PDF of higher SNR is given by the combined effect of APD internal noise and signal variations due to system vibrations. At lower SNRs, the APD internal noise dominates and the distribution approaches to Gaussian.

Overall, Fig. 7 shows the robustness and adaptability of the method; as it is capable of working with low input power and provides sufficient signal quality improvement with a broad range of received power. This dynamic range allows its use with a variety of Gigabit reception system and modulation formats. (a)

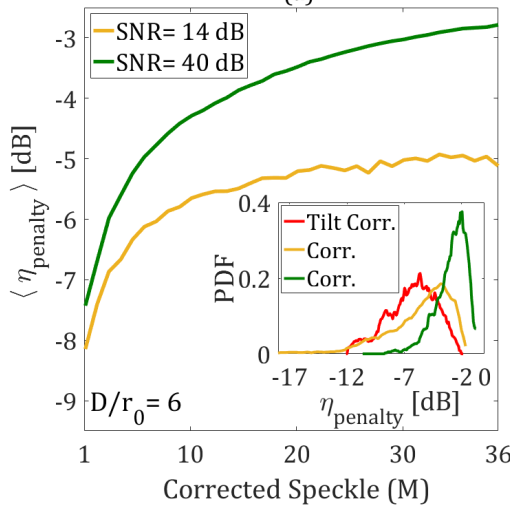

(b)

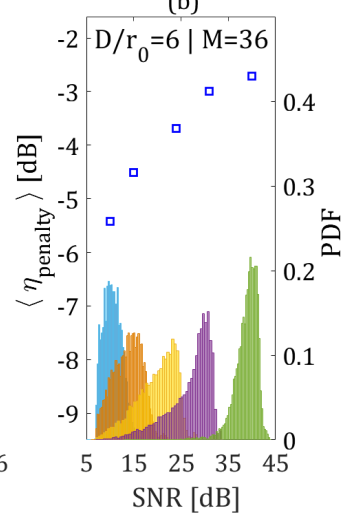

Fig. 7. (a) Coupling efficiency penalty vs. number of corrected speckles. The Inset shows the PDFs for the final coupling. (b) Evolution of the coupled signal and SNR distribution.

Figure 8 (a) shows the performance of the method in a range of weak $D / r_{0}=1$ to strong $D / r_{0}=6$ turbulence conditions. Stronger turbulence is not tested, limited by the maximum DM stroke in the illuminated area and resolution limits of the SLM, 
above which artifacts and low efficiency occur [21]. The tilt correction uses the center of gravity of the focal spots. Along with some residual system aberrations, it results in additional losses, especially in scenarios of low turbulence. The curves show a close matching between the experimental and numerical results alongside the tested scenarios. We noticed an important improvement in the mean and variance (inset plots) of the signal when the fields are corrected with $M=36$ (106 iterations) and $M=20$ (58 iterations). A nearly half-reduction in bandwidth utilization comes at the expense of a minimal penalty of $0.4 \mathrm{~dB}$ in the signal mean and a slight increase in the variance. All in all, the performance shows stability in a wide range of turbulence conditions and reduced bandwidth utilization, both necessary properties for the potential use of this technique in LEO downlink scenarios. The long-term point spread function can be visualized by performing the ensemble of the corrected intensity patterns $\langle\boldsymbol{I}\rangle$ over a set of $N=500$ captured focal images [22],

$$
\langle\boldsymbol{I}\rangle=\frac{1}{N} \sum_{m=1}^{N} \boldsymbol{I}_{m}
$$

The result is shown in Fig. 8 (b), and the calculation is performed with Eq. (13) before and after the correction of $M=20$ speckles per field. The beam intensity wave-fronts shown in the insets highlight beam focusing improvement.
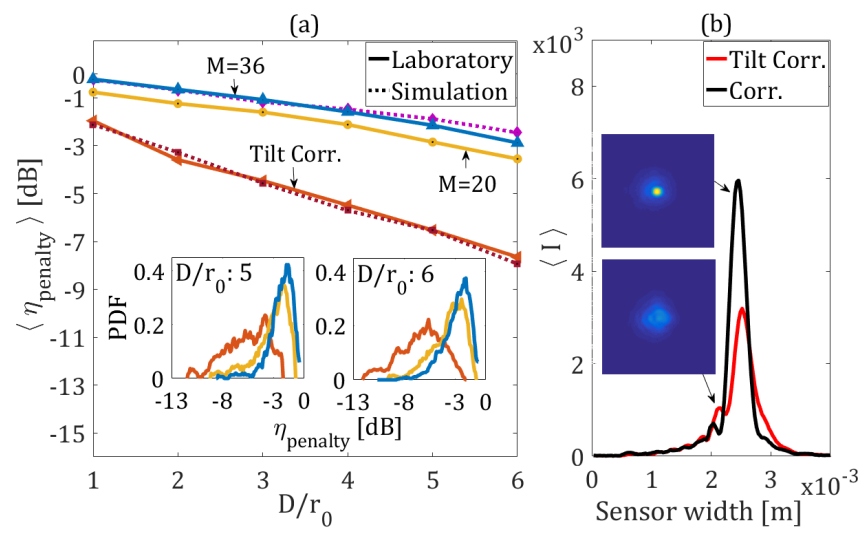

Fig. 8. (a) Overall performance for different turbulent scenarios. The inset shows the signal distribution. (b) Cumulative normalized intensity before and after correction.

\section{CONCLUSIONS}

Summarizing, to deal with the problem of phase compensation in laser downlinks affected by strong turbulence, we have presented an alternative iterative solution that uses focal intensity images to reduce the bandwidth utilization, while improving the quality of the received signal. This technique works by iteratively updating the phases of individual speckles to maximize the received power coupled into a single-mode fiber.

We have experimentally verified that this method is capable of dealing with weak to strong turbulence conditions, improving the mean coupling efficiency and reducing the signal fluctuations with less than 60 power measurements. We have also discussed the impact of the noise in the performance and concluded that the method show robustness, adaptability, and can deliver sufficient signal improvement in a wide range of turbulence conditions and received power. With this, we have highlighted its versatility regarding its use with a variety of gigabit reception schemes. The good matching between the laboratory results and numerical analysis allow us projecting the potential improvements of this technique with enhanced hardware characteristics.

Further research is required towards the dynamic and realtime performance analysis of the method. Once demonstrated its lower loop bandwidth requirements, a proper control algorithm needs to be developed to test the concept in a real-time regime. Likewise, improvements in the optical system design can be made to reduce aberrations. Finally, a dedicated tilt-correction system for the communication fiber may be used to reduce the signal variance further.

\section{REFERENCES}

1. T. R. Ellis and J. D. Schmidt, "Wavefront sensor performance in strong turbulence with an extended beacon," in 2010 IEEE Aerospace Conference, (IEEE, 2010), pp. 1-10.

2. E. Anzuola, M. Segel, S. Gladysz, and K. Stein, "Performance of wavefront-sensorless adaptive optics using modal and zonal correction," in SPIE Optics in Atmospheric Propagation and Adaptive Systems XIX, , vol. 10002 SPIE, ed. (Edinburgh, United Kingdom, 2016), p. 100020J.

3. F. Moll and M. Knapek, "Free-space laser communications for satellite downlinks: Measurements of the atmospheric channel," in 62nd International Astronautical Congress, t. I. A. Federation, ed. (Cape Town, SA, 2010), pp. 1-9.

4. N. Perlot, M. Knapek, D. Giggenbach, J. Horwath, M. Brechtelsbauer, Y. Takayama, and T. Jono, "Results of the optical downlink experiment KIODO from OICETS satellite to optical ground station Oberpfaffenhofen (OGS-OP)," in Free-Space Laser Communication Technologies $X I X$ and Atmospheric Propagation of Electromagnetic Waves, , vol. 645704 (SPIE, San Jose, California, 2007).

5. H. Yang and X. Li, "Comparison of several stochastic parallel optimization algorithms for adaptive optics system without a wavefront sensor," Opt. Laser Technol. 43, 630-635 (2011).

6. M. A. Vorontsov, "Decoupled stochastic parallel gradient descent optimization for adaptive optics : integrated approach for wave-front sensor information fusion," J. Opt. Soc. Am. A 19, 356-368 (2002).

7. C. E. Carrizo, R. M. Calvo, and A. Belmonte, "Intensity-based adaptive optics with sequential optimization for laser communications," OPTICS EXPRESS 26, 16044-16053 (2018).

8. L. C. Andrews and R. L. Phillips, Laser Beam Propagation through Random Media second edition (SPIE, 2005).

9. M. Toyoshima, H. Takenaka, and Y. Takayama, "Atmospheric turbulence-induced fading channel model for space-to-ground laser communications links," Opt. Express 19, 15965 (2011).

10. Y. Dikmelik and F. M. Davidson, "Fiber-coupling efficiency for freespace optical communication through atmospheric turbulence," Appl. Opt. 44, 4946 (2005).

11. H. Takenaka, M. Toyoshima, and Y. Takayama, "Experimental verification of fiber-coupling efficiency for satellite-to-ground atmospheric laser downlinks," Opt. Express 20, 15301 (2012).

12. M. P. Cagigal and V. F. Canales, "Generalized Fried parameter after adaptive optics partial wave-front compensation," J. Opt. Soc. Am. A, Opt. image science, vision 17, 903-10 (2000).

13. P. J. Winzer and W. R. Leeb, "Fiber coupling efficiency for random light and its applications to lidar," Opt. Lett. 23, 986 (1998).

14. A. Belmonte, "Capacity of Coherent Laser Downlinks," Light. Technol. 32, 2128-2132 (2014).

15. A. Belmonte and J. M. Kahn, "Sequential optimization of adaptive arrays in coherent laser communications," J. Light. Technol. 31, 13831387 (2013).

16. N. A. Roddier, "Atmospheric wavefront simulation using Zernike polynomials," Opt. Eng. 29 (1990).

17. A. Natan, "2D fast peak finder,"

18. D. Giggenbach, P. Becker, R. Mata-calvo, C. Fuchs, Z. Sodnik, and I. Zayer, "Lunar Optical Communications Link ( LOCL ): Measurements 
of Received Power Fluctuations and Wavefront Quality," in ICSOS International Conference on Space Optical Systems and Applications, (Kobe, Japan, 2014).

19. D. Giggenbach and R. Mata-Calvo, "Sensitivity modeling of binary optical receivers," Appl. Opt. 54, 8254-8259 (2015).

20. P. Conroy, J. Surof, J. Poliak, and R. M. Calvo, "Demonstration of 40 GBaud intradyne transmission through worst-case atmospheric turbulence conditions for geostationary satellite uplink," Appl. Opt. 57, 5095-5101 (2018).

21. I. a. Litvin, L. Burger, M. P. D. Gama, A. Mathye, and A. Forbes, "Laser beam shaping limitations for laboratory simulation of turbulence using a phase-only spatial light modulator," Proc. SPIE 6663, 66630R-1 (2007).

22. J. W. Goodman, Speckle Phenomena in Optics: Theory and Applications (Roberts and Company, 2007). 